\title{
ASSESSING THE ACCURACY OF STATISTICAL PROPERTIES EXTRACTED FROM A LIMITED NUMBER OF DEVICE UNDER TEST FOR TIME DEPENDENT VARIATIONS
}

\author{
J. F. Zhang, M. Duan, Z. Ji, and W. D. Zhang \\ Department of Electronics and Electrical Engineering, Liverpool John Moores University, \\ Byrom Street, Liverpool L3 3AF, UK \\ E-mail: j.f.zhang@ljmu.ac.uk
}

\begin{abstract}
As device sizes scale down, device variations scale up. There are two types of device-to-device variations (DDV): as-fabricated or time-zero DDV and the time dependent variations (TDV). Even if two nano-scaled devices were identical at time-zero, they would be different after stresses and result in TDV, since the defect generation and charging-discharging are stochastic. To characterize TDV, statistical properties, such as the mean value and standard deviation, are extracted from tests. Their accuracy improves as the number of device under tests (DUTs) increases. Ageing is time consuming and the typical DUTs used are in the range of tens to hundreds. There is little information on the accuracy of the statistical properties extracted from such a limited DUTs and the objective of this paper is to propose a methodology to assess it. Based on the defect-centric model, the accuracy with a specific confidence level is evaluated for a given number of DUTs and a stress level.
\end{abstract}

\section{INTRODUCTION}

As CMOS technologies enter the nano-meter range, device-to-device variations (DDV) become a challenge for circuit design and optimization [1-12]. There are two types of DDV: the as-fabricated DDV at time zero and the time dependent DDV. The as-fabricated DDV has a number of origins, such as random discrete dopant, line edge roughness, gate work function variation, and FIN size variation etc [1]. They have been extensively investigated. This work focuses on the time dependent DDV (TDV). Under electrical stresses, transistors age through charging/discharging either as-grown traps or generated traps in gate dielectric [13-20]. Even though two devices can be identical at time zero, they become different after electrical stresses, because the defect generation and the charge-discharge of traps in the gate dielectric are stochastic [1-3]. One example is given in Fig. 1, where the ageing of two devices is clearly different under the same stress biases [9].

To characterize DDV, the common practice is to repeat the same test on multiple devices and extract the statistical properties, such as the mean value and standard deviation [1-12]. For as-fabricated DDV, the measurement is fast and a large number of devices can be used in a practical test time. For TDVs, however, device ageing can be a time consuming process and the number of Device Under Tests (DUTs) used is limited. While the DUTs can reach the order of $\sim 10^{5}$ [4] for relative short time such as $1000 \mathrm{sec}$, they are often only in the range of tens to hundreds for longer stress time [2-12]. As the accuracy of the statistical properties always improve with number of DUTs, the question is how accurate they are when extracted from a limited number of DUTs. In this work, we will develop a methodology to assess this accuracy, based on the defect centric model [2-4].

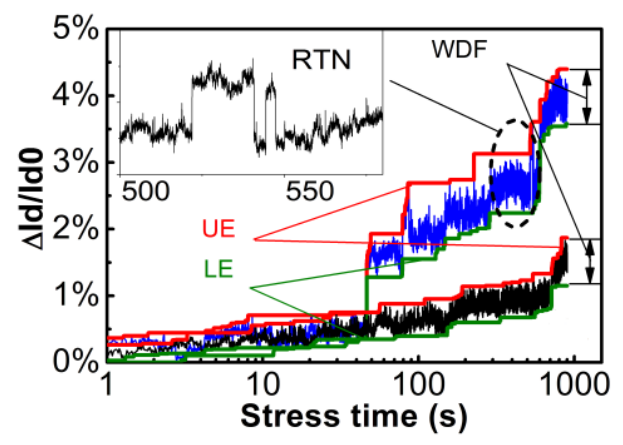

Fig. 1 An example of time dependent device-to-device variation (TDV). Two devices of the same size were subjected to the same electrical stress. Their ageing is clearly different [9].

\section{DEFECT CENTRIC MODEL}

The defect centric model $[2,3]$ is based on two assumptions. One of them is that the number of traps in gate dielectric per device is random and follows the Poisson distribution. The other is that the impact of a trap on a device in term of parameter shift, such as threshold voltage shift $\Delta \mathrm{Vth}$, follows an exponential distribution. This leads to a combined distribution function of $[2,3,9]$, 
$H_{\eta, N t}(\Delta V t h)=\sum_{k=0}^{\infty} \frac{e^{-N t} N t^{k}}{k !}\left[1-\frac{k}{k !} \Gamma\left(k, \frac{\Delta V t h}{\eta}\right)\right]$

where $\mathrm{H}$ is the cumulative distribution, $\Gamma$ is the Gamma function, $\eta$ is the average impact of one trap on the device and $\mathrm{Nt}$ is the average number of traps per device. They are related to the mean, $\mu$, and standard deviation, $\sigma$, by:

$$
N t=\frac{2 \mu^{2}}{\sigma^{2}},
$$

and

$$
\eta=\frac{\sigma^{2}}{2 \mu}
$$

Once the $\mu$ and $\sigma$ is obtained from the test data, one can evaluate Nt and $\eta$ by eqs. (2) and (3) and in turn the statistical distribution by eq. (1).

\section{THE MODEL VERSUS TEST DATA}

For the TDVs induced by the bias temperature instabilities, the defect centric model has been verified based on extensive amount of tests: 92,000 DUTs from 4000 process lots [4].
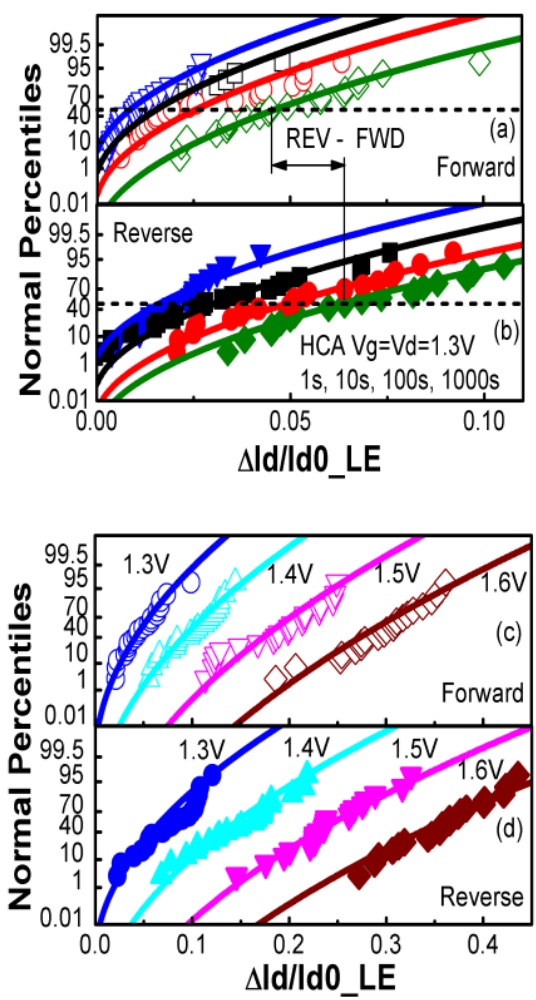

Fig. 2 An example of good agreement between the test data (symbols) and the defect-centric model (lines). 'Forward' means the source and drain for stress and measurement are the same, while 'Reverse' means that they were swapped after stresses [9].
For the TDVs induced by hot carrier ageing (HCA), there is also a good agreement between the model and the test data, which were taken after different stress times in Figs. 2(a) and 2(b) and different stress biases in Figs. 2(c) and $2(\mathrm{~d})$

The model predicts that standard deviation is related to the mean by a power law,

$$
\sigma=\sqrt{2 \eta \mu}
$$

This again agrees with the test data in Fig. 3 [9].

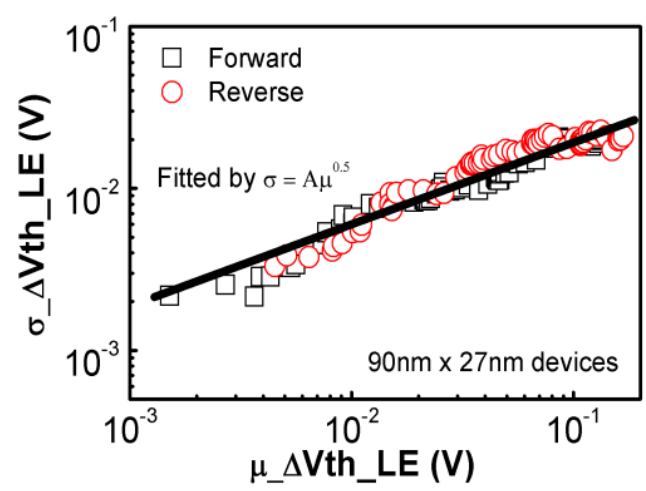

Fig. 3 The relation between standard deviation and the mean follows the prediction by eq. (4) well [9].

\section{METHODOLOGY}

For a given $\mathrm{Nt}$ and $\eta$, one can construct hypothetic devices with the number of traps in each device, nt, determined by the Poisson distribution and the threshold voltage shift induced by a trap, $\Delta \mathrm{Vth}, \mathrm{i}$, obtained by the exponential distribution. The total $\Delta \mathrm{V}$ th of this device is the sum of each-trap induced shift,

$$
\Delta V t h=\sum_{i=1}^{n t} \Delta V t h, i
$$

These hypothetic devices allow one to simulate the experiments that use a limited number of DUTs for extracting the $\mu$ and $\sigma$. For example, one test engineer used $X$ DUTs can be simulated by generating $X$ hypothetic devices for the 'Test 1 ' in Fig. 4. The X $\Delta$ Vth for these devices is then used to evaluate $\mu$ and $\sigma$, which corresponds to one point in Figs. 5(a) and (b), respectively.

Now we assume that a different test engineer is doing the same test by using X DUTs again. These X DUTs of course can be different from those used by the previous engineer and we imitate this by randomly generating the second set of X DUTs, labeled as 'Test 2' in Fig. 4. This will produce another point for $\mu$ and $\sigma$ in 5 (a) and (b), respectively. 
Repeating the same simulation for a sufficiently larger number of sets, i.e. the $\mathrm{M}$ in Fig. 4, one can obtain the distribution of extracted $\mu$ and $\sigma$ for a given X DUTs, as shown in Fig. 5. They can then be compared with the true $\mu$ and $\sigma$ that is evaluated by eqs. (2) and (3) for the given $\mathrm{Nt}$ and $\eta$ used to generate the hypothetic devices. At a given level of confidence, the accuracy of $\mu$ and $\sigma$ for a specific DUTs can be evaluated, as shown in Fig. 7 and Fig. 8.

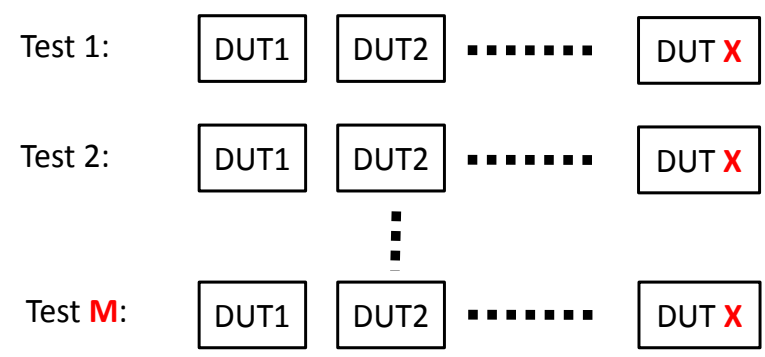

Fig. 4 An illustration of statistical tests: In a hypothetic Test 1 , engineer 1 used XDUTs for extracting the $\mu$ and $\sigma$ of TDV. In test 2, engineer 2 also used X DUTs, but will obtain different $\mu$ and $\sigma$, because a different set of devices were used [10].

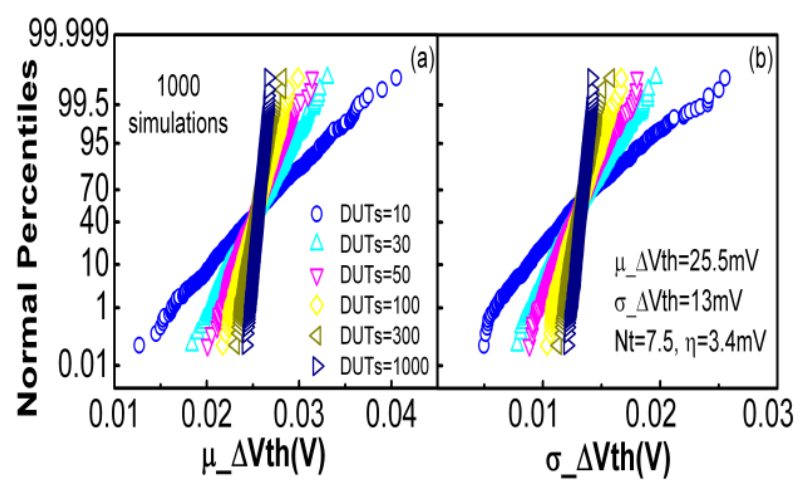

Fig. 5 The $\mu(a)$ and $\sigma(b)$ extracted for different number of DUTs ( $X$ in Fig. 4). For a given $X$, the tests were repeated 1000 times $(M=1000$ in Fig. 4) [10].

\section{APPLICATION EXAMPLES}

Device lifetime is typically defined as the time for $\Delta V$ th reaching $25 \sim 50 \mathrm{mV}$. The average $\Delta \mathrm{Vth}$ induced by one trap, $\eta$, is $\sim 3.4 \mathrm{mV}$, evaluated from the slope of the fitted line in Fig. 3. This gives an average number of traps per device, $\mathrm{Nt}=\Delta \mathrm{Vth} / \eta$, of $7 \sim 15$.

Fig. 5 shows the distribution for $\mathrm{Nt}=7.5$, when the same set of tests were repeated 1000 times, i.e. $M=1000$ in Fig. 4. As expected, the statistical spread is larger for smaller DUTs in both $\mu$ and $\sigma$.

The number of DUTs is not the only parameter affecting the spread. Fig. 6 shows that Nt also has an impact. The spread reduces for larger $\mathrm{Nt}$, since a larger $\mathrm{Nt}$ averages out the individual effect of a trap on a device.

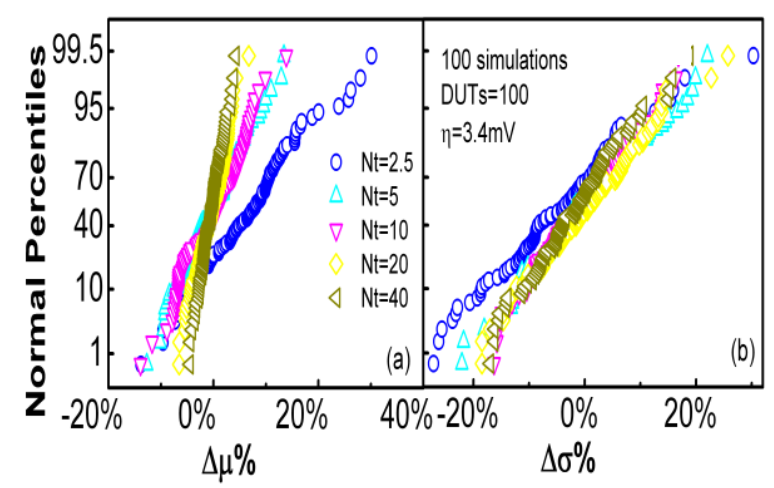

Fig. 6 The impact of the average number of traps, Nt, per DUT on the $\mu(a)$ and $\sigma$ (b) extracted for DUTs $=100$ when the tests were repeated 1000 times (M=1000 in Fig. 4) [10].

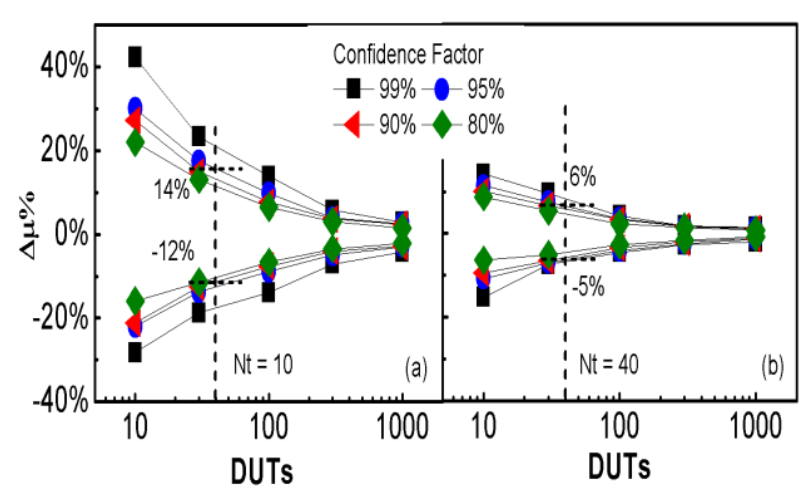

Fig. 7 The dependence of the accuracy of mean value, $\mu$, on the number of DUTs used in a test for $N t=10(a)$ and $N t=40$ (b). The accuracy with a 95\% confidence is marked out for 40 devices [10].

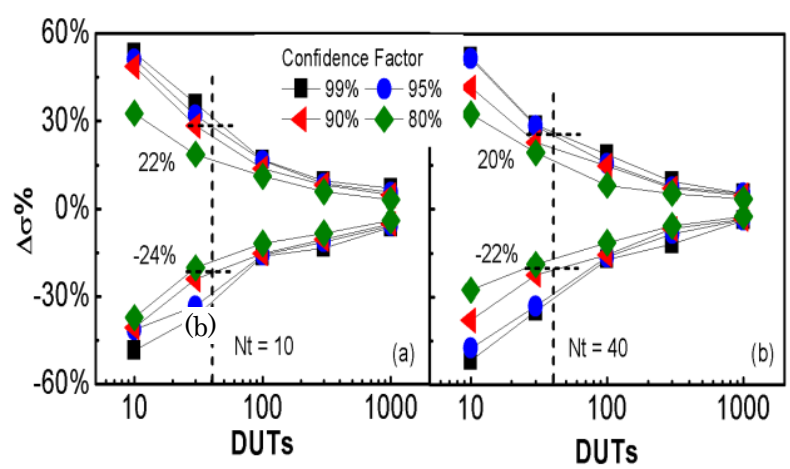

Fig. 8 The dependence of the accuracy of standard deviation, $\sigma$, on the number of DUTs used in a test for $N t=10$ (a) and $N t=40$ (b). The accuracy with a 95\% confidence is marked out for 40 devices [10]. 
Figs. 7(a) and (b) shows the accuracy of extracted $\mu$ for $\mathrm{Nt}=10$ and 40 , respectively. For $\mathrm{Nt}=10, \mu$ has an accuracy within $\pm 14 \%$ with a $95 \%$ confidence when $\mathrm{X}=40$. It is improved to $\pm 6 \%$ when $\mathrm{Nt}=40$. If 1000 DUTs were used, the accuracy will be improved to $\pm 2.6 \%$ for $\mathrm{N}=10$ and $\pm 1.3 \%$ for $\mathrm{Nt}=40$.

For the same DUTs and Nt, the accuracy in $\sigma$ is less. Figs. 8(a) and (b) shows the accuracy of extracted $\sigma$ for $\mathrm{Nt}=10$ and 40, respectively. For $\mathrm{Nt}=10, \sigma$ only has an accuracy within $\pm 24 \%$ with a $95 \%$ confidence when $\mathrm{X}=40$. It is improved only to $\pm 22 \%$ when $\mathrm{Nt}=40$. If 1000 DUTs were used, the accuracy will be improved to $\pm 5 \%$ for $\mathrm{Nt}=10$.

\section{CONCLUSIONS}

Defect generation and charge-discharge of traps in gate dielectric are stochastic processes. This induces a time dependent device-to-device variations (TDVs). In this work, we propose a methodology for assessing the accuracy of the statistical properties of TDVs extracted from a limited number of DUTs, based on the defect-centric model. An increase of either the number of DUTs or the number of average traps per device improves the accuracy of mean and standard deviation. When the average number of traps per device $(\sim 10)$ corresponds to typical definition for device lifetime, an accuracy for 40 DUTs is around $14 \%$ and $24 \%$ for the mean and standard deviation, respectively. They are improved to $2.6 \%$ and 5\%, when 1000 DUTs are used.

\section{ACKNOWLEDGEMENTS}

The authors thank D. Vigar of Qualcomm for supplying the test samples and B. Kaczer of IMEC, Belgium, and A. Asenov of Glasgow University for discussions. This work is supported by the EPSRC of UK under the grant no. EP/L010607/1.

\section{REFERENCES}

[1] A. Asenov, B. Cheng, D. Dideban, U. Kovac, N. Moezi, C. Millar, G. Roy, A. R. Brown, and S. Roy, Proc. IEEE Custom Integr. Circuits Conf., 2010, pp. $1-8$.

[2] B. Kaczer, T. Grasser, P. J. Roussel, J. Franco, R. Degraeve, L. A. Ragnarsson, E. Simoen, G. Groeseneken, and H. Reisinger, Proc. Int. Rel. Phys. Symp., 2010, pp. 26-32.

[3] L. M. Procel, F. Crupi, J. Franco, L. Trojman, and B. Kaczer, IEEE Elec. Dev. Lett., vol. 35, 2014, pp. 1167-1169.

[4] C. Prasad, M. Agostinelli, J. Hicks, S. Ramey, C. Auth, K. Mistry, S. Natarajan, P. Packan, I. Post, S. Bodapati, M. Giles, S. Gupta, S. Mudanai, and K. Kuhn, Proc. Int. Rel. Phys. Symp., 2014, pp. 6A.5.1-6A.5.7.
[5] M. Duan, J. F. Zhang, Z. Ji, W. Zhang, B. Kaczer, T. Schram, R. Ritzenthaler, G. Groeseneken, and A. Asenov, IEEE Trans. Elec. Dev., vol. 60, 2013, pp. 2505-2511.

[6] M. Duan, J. F. Zhang, Z. Ji, W. D. Zhang, B. Kaczer, T. Schram, R. Ritzenthaler, G. Groeseneken, and A. Asenov, IEEE Trans. Elec. Dev., vol. 61, 2014, pp. 3081-3089.

[7] M. Duan, J. F. Zhang, Z. Ji, J. G. Ma, W. Zhang, B. Kaczer, T. Schram, R. Ritzenthaler, G. Groeseneken, and A. Asenov, Proc. IEDM, 2013, pp. 774-777.

[8] M. Duan, J. F. Zhang, Z. Ji, W. Zhang, B. Kaczer, T. Schram, R. Ritzenthaler, A. Thean, G. Groeseneken, and A. Asenov, Proc of IEEE VLSI Tech. Symp., 2014, pp. 74-75.

[9] M. Duan, J. F. Zhang, A. Manut, Z. Ji, W. Zhang, A. Asenov, L. Gerrer, D. Reid, H. Razaidi, D. Vigar, V. Chandra, R. Aitken, B. Kaczer, and G. Groeseneken, Proc. IEDM, 2015, pp. 547-550.

[10] M. Duan, J. F. Zhang, Z. Ji, W. Zhang, B. Kaczer, and A. Asenov, IEEE Trans. Elec. Dev., vol. 64, 2017, pp. 2478-2484.

[11] R. Gao, Z. Ji, S. M. Hatta, J. F. Zhang, J. Franco, B. Kaczer, W. Zhang, M. Duan, S. De Gendt, D. Linten, G. Groeseneken, J. Bi and M. Liu, Proc. IEDM, 2016, pp. 778-781.

[12] R. Gao, Z. Ji, A. B. Manut, J. F. Zhang, J. Franco, S. W. M. Hatta, W. D. Zhang, B. Kaczer, D. Linten, and G. Groeseneken, IEEE Trans. Elec. Dev., vol. 64, 2017, pp. 4011-4017.

[13] M. H. Chang, J. F. Zhang, and W. D. Zhang, IEEE Trans. Elec. Dev., vol. 53, 2006, pp. 1347-1354.

[14] J. F. Zhang, I. S. Al-kofahi, and G. Groeseneken, J. Appl. Phys., vol. 83, 1998, pp.843-850.

[15] J. F. Zhang, C. Z. Zhao, G. Groeseneken, and R. Degraeve, J. Appl. Phys., vol. 93, 2003, pp.6107-6116.

[16] J. F. Zhang, M. H. Chang, Z. Ji, L. Lin, I. Ferain, G. Groeseneken, L. Pantisano, S. De Gendt, and M. M. Heyns, IEEE Elec. Dev. Lett., vol. 29, 2008, pp.1360-1363.

[17] C. Z. Zhao and J. F. Zhang, J. Appl. Phys., vol. 97, art no. 073703.

[18] M. Duan, J. F. Zhang, Z. Ji, W. Zhang, B. Kaczer, S. De Gendt, and G. Groeseneken, IEEE Elec. Dev. Lett., vol. 33, 2012, pp. 480-482.

[19] J. F. Zhang, C. Z. Zhao, G. Groeseneken, R. Degraeve, J. N. Ellis, and C. D. Beech, Solid-State Electronics, vol.46, 2002, pp.1839-1847.

[20] Z. Ji, S. F. W. M. Hatta, J. F. Zhang, J. G. Ma, W. Zhang, N. Soin, B. Kaczer, S. De Gendt, and G. Groeseneken, Proc. IEDM, 2013, pp. 413-416. 